\title{
Systematic Review of the Risk of Permanent Alopecia with Docetaxel Treatment for Breast Cancer
}

\section{Shirin Nezafat Namini ${ }^{*}$}

Department of Clinical Oncology, St James Teaching Hospital, Beckett Street, England

*Corresponding author: Shirin Nezafat Namini, Department of Clinical Oncology, St James Teaching Hospital, Beckett Street, England, Tel: 447787755185; E-mail: shirinnamini@hotmail.co.uk

Rec date: Mar 09, 2016; Acc date: Aug 14, 2016; Pub date: Aug 17, 2016

Copyright: (c) 2016 Namini SN. This is an open-access article distributed under the terms of the Creative Commons Attribution License, which permits unrestricted use, distribution, and reproduction in any medium, provided the original author and source are credited.

\begin{abstract}
Aim: The most commonly reported adverse reactions of docetaxel are neutropenia, anaemia, alopecia, nausea, vomiting, stomatitis, diarrhoea and asthenia. Alopecia is a distressing side-effect of cancer treatment and can have a significant negative impact on self-esteem and quality of life. The incidence of permanent alopecia with docetaxel is unclear. We reviewed available evidence relating to permanent alopecia associated with docetaxel given for the treatment of breast cancer.
\end{abstract}

Method: A full literature search was performed using Medline with key words docetaxel and alopecia. Studies were included if they reported the use of docetaxel either alone or in combination regimens in breast cancer and provided information on permanent alopecia.

Results: In total three papers were found. Tallon et al. reported a case of permanent alopecia in a 72-year-old caucasian female who was treated with 3 weekly adjuvant carboplatin (AUC6 $650 \mathrm{mg}$ ), docetaxel $\left(75 \mathrm{mg} / \mathrm{m}^{2}\right)$ and trastuzumab $(4 \mathrm{mg} / \mathrm{kg})$ for 6 cycles. This was followed by a year of 3 weekly trastuzumab. After her first cycle carboplatin was removed from the regimen because of severe neutropenia. She experienced scalp hair loss 2 weeks into treatment and this persisted at 13 months after completion of treatment with only minimal re-growth. A punch biopsy showed a reduction in hair follicles. She had no previous history of alopecia but was treated for hypothyroidism.

Keywords: Breast cancer; Permanent alopecia; Docetaxel

\section{Introduction}

The most commonly reported adverse reactions of docetaxel are neutropenia, anaemia, alopecia, nausea, vomiting, stomatitis, diarrhoea and asthenia [1]. Scalp, eyebrow hair, eyelashes, axillary and pubic hair is routinely lost and this is usually reversible and hair regrows after 3-6 months. We therefore routinely counsel patients about the risk of temporary hair loss during their chemotherapy treatment. However, in recent years there has been increasing anecdotal evidence regarding the incidence of permanent alopecia following treatment with chemotherapy. This most commonly occurs with high dose myeloablative regimens, bleomycin and cyclophosphamide but there have also been reports of permanent alopecia associated with docetaxel regimens used in breast cancer. The incidence of permanent alopecia with docetaxel is unclear $[2,3]$.

\section{Case Report}

Prevezas et al. [4] reported a 58-year-old female patient who developed diffuse and irreversible alopecia after being treated with six cycles of docetaxel $75 \mathrm{mg} / \mathrm{m}^{2}$ every 3 weeks for locally recurrent breast cancer. There was diffuse alopecia with characteristics of androgenetic hair loss and an absence of scalp fibrosis. Scalp biopsy showed reduced hair follicle density with an increased number of vellus hairs. There was a mild dermal lymphocytic infiltrate, but no peribulbar inflammation or fibrosis was documented. Treatment with topical minoxidil produced slight improvement which was secondary to an increase of the volume of the persisting hair.

A series of 20 women treated for breast cancer with sequential fluorouracil/epirubicin/cyclophosphamide and docetaxel between 2007 and 2011 who developed permanent alopecia was reported by Kluger et al. [5] Permanent alopecia (defined as present 6 months or longer after completing chemotherapy occurred predominantly over the crown in an androgenic like pattern with eyelash and eyebrow loss. 18 were under-going neoadjuvant or adjuvant chemotherapy and 2 were being treated for relapsed disease.

Most patients received epirubicin $100 \mathrm{mg} / \mathrm{m}^{2}$ cyclophosphamide $500 \mathrm{mg} / \mathrm{m}^{2}$ and 5 -fluorouracil $500 \mathrm{mg} / \mathrm{m}^{2}$ every 3 weeks for 3 cycles followed by docetaxel $100 \mathrm{mg} / \mathrm{m}^{2} 3$ weekly for 3 or 4 cycles. Other potential causes of alopecia were excluded and biopsies were either normal or displayed an androgenic like pattern. They also assessed the impact of alopecia on quality of life using mean DLQI (Dermatology Life Quality Index) score. Seven patients showed severe impairment and the overall mean score was well below that of a healthy population.

In the TAX-316 study [6], which was a multicentre phase III randomized trial comparing docetaxel in combination with doxorubicin and cyclophosphamide (TAC) versus 5-fluorouracil in combination with doxorubicin and cyclophosphamide (FAC) as adjuvant treatment of operable breast cancer patients with positive axillary lymph nodes. Alopecia persisting into the follow-up period after the end of chemotherapy was reported in 687 TAC patients and 645 FAC patients. At the end of the follow-up period, alopecia was 
Citation: Namini SN (2016) Systematic Review of the Risk of Permanent Alopecia with Docetaxel Treatment for Breast Cancer. J Clin Case Rep 6: 851. doi:10.4172/2165-7920.1000851

Page 2 of 2

observed to be on-going in 29 TAC patients (4.2\%) and 16 FAC patients $(2.4 \%)$.

\section{Discussion}

This review would suggest that there is a risk of permanent alopecia with docetaxel. However, the data are very limited and dependent on small sample sizes. In addition, it is not known whether the severity of alopecia is related to the dose of docetaxel given or by other drugs often given in combination. Despite this there does seem to be a direct link between docetaxel and permanent alopecia associated with a reduction in total number of follicles without inflammation or fibrosis. Proposed hypotheses for this have included toxic damage to stem cells of the hair matrix, disturbance of signalling pathways to the secondary germ cell or endocrine disturbances [4].

Research into preventative measures of permanent and temporary alopecia is needed. Scalp cooling is commonly employed and is commonly effective in the context of temporary alopecia, but there are no useful data concerning permanent alopecia [7].

Alopecia is a distressing side-effect of cancer treatment and can have a significant negative impact on self-esteem and quality of life. Available patient literature does not specify that there is a small risk of permanent alopecia with docetaxel and further research is needed to identify its incidence and pathophysiology and to identify possible preventative interventions.

\section{Conflicts of interest}

No conflicts of interest to declare

\section{References}

1. Palamaras I, Misciali C, Vincenzi C, Robles WS, Tosti A (2011) Permanent chemotherapy induced alopecia: a review. J Am Acad Dermatol 64: 604-606.

2. Miteva M, Miscialli C, Fanti PA, Vincenzi C, Romanelli P, et al. (2011) Permanent alopecia after systemic chemotherapy: a clinicopathological of 10 cases. Am J Dermatopathol 33: 345-350.

3. Tallon B, Blanchard E, Goldberb LJ (2010) Permanent chemotherapy induced alopecia: Case report and review of the literature. J Am Acad Dermatol 63: 333-336.

4. Prevezas C, Matard B, Pinquier L, Reygagne P (2009) Irreversible and severe alopecia following docetaxel or paclitaxel cytotoxic therapy for breast cancer. Br J Dermatol 160: 883-885.

5. Kluger N, Jacot W, Frouin E, Rigau V, Poujol S (2012) Permanent alopecia related to breast cancer chemotherapy by sequential flurorouracil/ epirubicin/cyclophosphosamide and docetaxel: a prospective study of 20 patients. Ann Oncol 23: 2879-2884.

6. Martin M, Pienkowski T, Mackey J, Pawlicki M, Guastalla JP, et al. (2005) Adjuvant Docetaxel for Node-Positive Breast Cancer. N Engl J Med 352: 2302-2313.

7. Katsimbri P, Bamias A, Pavlidis N (1997) Prevention of chemotherapyinduced alopecia using an effective scalp cooling system. Eur J Cancer 33: 766-771. 